\title{
Matutinidad-vespertinidad y ansiedad rasgo en adolescentes
}

\author{
Ma . José Collado-Mateo, Juan Francisco Díaz-Morales*, Cristina Escribano y Pedro Delgado \\ Universidad Complutense de Madrid, España
}

\begin{abstract}
Resumen: El objetivo de este trabajo fue estudiar la relación entre la matutinidad-vespertinidad y la ansiedad rasgo en adolescentes. La muestra estaba formada por 638 adolescentes de 12 a 14 años. Se midió la matutinidadvespertinidad mediante la Escala de Matutinidad-Vespertinidad para Niños, MESC (Carskadon, Vieira y Acebo, 1993) y la ansiedad rasgo mediante el Inventario de Ansiedad Estado-Rasgo para niños, STAIC (Spielberger, Edwards, Lushene, Montuori y Platzek, 1973; Spielberger, Edwards y Lushene, 1990). Los resultados indicaron que las chicas eran más vespertinas, que la vespertinidad aumentaba con la edad y una relación negativa entre matutinidad y ansiedad rasgo. Aquellos adolescentes más vespertinos obtuvieron una mayor ansiedad rasgo, posiblemente debido a un mayor desajuste entre los ritmos biológicos y sociales (horarios escolares matutinos).

Palabras clave: Matutinidad-vespertinidad; ansiedad rasgo; sexo; adolescencia.
\end{abstract}

\section{Introducción}

La Matutinidad-Vespertinidad (M-V) es una dimensión de diferenciación individual basada en los ritmos circadianos que hace referencia a las diferencias individuales en la preferencia por realizar las actividades durante la mañana o la tarde (Carskadon, Vieira y Acebo, 1993). Las personas matutinas están más sincronizadas con el ciclo luz/oscuridad y, en general, alcanzan el pico máximo o acrofase en los ritmos biológicos (i.e. temperatura, cortisol o melatonina) y psicológicos (i.e. atención o memoria) entre 1.5 y 3 horas antes que las vespertinas (Adan, Natale y Caci, 2008; Tankova, Adan y Buela-Casal, 1994).

Los cronotipos difieren tanto en sus características de personalidad como en sus condiciones de salud. Las personas matutinas se caracterizan por un estilo de personalidad analítico y conformista (Díaz-Morales, 2007), mientras que los vespertinos muestran un estilo de personalidad sintético y creativo (Fabbri, Antonietti, Giorgetti, Tonetti y Natale, 2007). En adolescentes la matutinidad se ha relacionado con persistencia y cooperación y la vespertinidad con búsqueda de sensaciones y auto-trascendencia (Randler y Saliger, 2011). Respecto a la salud, los vespertinos manifiestan con mayor frecuencia una peor salud física y psicológica (Randler, 2011; Delgado, Díaz-Morales, Escribano, Collado y Randler, 2012), hábitos de alimentación y sueño irregulares (Monk, Buysse, Potts, DeGrazia y Kupfer, 2004), más síntomas pre-menstruales (Negriff y Dorn, 2009), peor ajuste y rendimiento escolar (Escribano, Díaz-Morales, Delgado y Collado, 2012), problemas de atención, conductas delictivas y agresivas (Gau et al., 2007), consumo de drogas e intoxicaciones (Adan, 2010; Andershed, 2005), inestabilidad emocio-

* Dirección para correspondencia [Correspondence address]: Juan Francisco Díaz-Morales. Departamento Psicología Diferencial y del Trabajo, Facultad de Psicología, Universidad Complutense de Madrid, Campus de Somosaguas, s/n, 28223, Madrid (España). E-mail: juanfcodiaz@psi.ucm.es
Title: Morningness-eveningness and anxiety trait among adolescents. Abstract: The relationship between morningness-eveningness and anxiety trait among adolescents was studied. Six hundred thirty eight adolescents between 12 and 14 years old participated in this study. Morningnesseveningness was measured by the Morningness-Eveningness Scale for Children, MESC (Carskadon, Vieira \& Acebo, 1993) and anxiety trait was measured by the State-Trait Anxiety Inventory for Children, STAIC (Spielberger, Edwards, Lushene, Montuori \& Platzek, 1973; Spielberger, Edwards \& Lushene, 1990). Results showed higher eveningness among girls, eveningness increased with age and anxiety trait was higher as morningness diminished. Therefore, evening-oriented adolescents claimed higher anxiety trait, which was waited since they would have a greater misalignment between both biological and social rhythms (school morning schedules).

Key words: Morningness-eveningness; anxiety trait; gender; adolescence.

nal (Giannotti, Cortesi, Sebastiani y Ottaviano, 2002), peores conocimientos en higiene de sueño (Adan, Fabbri, Natale y Prat, 2006; Díaz-Morales, Delgado, Escribano, Collado y Randler, 2011; Digdon, 2010) y síntomas de ansiedad y depresión (Gau et al., 2007; Giannotti et al., 2002).

En la adolescencia la creciente tendencia hacia la vespertinidad respecto a la niñez provoca un importante desajuste respecto a los horarios escolares matutinos (Carskadon et al., 1993). Este tipo de desajuste entre los ritmos biológicos y sociales se ha descrito como jetlag social (Wittmann, Dinich, Merrow y Roenneberg, 2006) y afecta a la salud y el bienestar (Benca et al., 2009; Kalsbeek et al., 2011; Kohyama, 2009; Mecacci y Rocchetti, 1998). En este sentido, una mayor ansiedad rasgo podría considerarse una consecuencia del desajuste permanente o crónico entre los ritmos biológicos y sociales, siendo los vespertinos los que muestran mayor ansiedad en una sociedad preferentemente matutina (DíazMorales y Sánchez-López, 2008).

El estudio de los distintos aspectos relacionados con la ansiedad es especialmente relevante puesto que estos trastornos son los más prevalentes en población general (Alonso et al., 2004). Además, la ansiedad puede considerarse también como un rasgo de personalidad (Spielberger, Edwards y Lushene, 1990). En la adolescencia la ansiedad puede interferir con el desarrollo y la maduración interpersonal, el rendimiento académico y las relaciones familiares dando lugar a otros trastornos durante la edad adulta (Sandín, 1997).

Distintos autores subrayan la relevancia del sexo/género en los estudios sobre personalidad y ritmos circadianos (Díaz-Morales y Sánchez-López, 2008; Muro, Gomà-iFreixanet y Adan, 2009), así como del cronotipo intermedio que representa aproximadamente al $60 \%$ de la población (Adan et al., 2008). Dado que la ansiedad es más prevalente en las mujeres y que aparece desde edades tempranas, parece pertinente estudiar si la $\mathrm{M}-\mathrm{V}$ se asocia con la ansiedad en ambos sexos en el inicio de la adolescencia. Los escasos estudios previos realizados han encontrado mayor ansiedad en 
adultos vespertinos (Díaz-Morales y Sánchez-López, 2008) y en mujeres adolescentes (Pabst, Negriff, Dorn, Susman y Huang, 2009).

En este trabajo se analiza la relación entre M-V y la ansiedad rasgo en adolescentes considerando el sexo y la edad. Se espera una menor matutinidad entre las chicas y a medida que los adolescentes tienen más edad, así como una mayor ansiedad rasgo en las chicas y a medida que disminuye la matutinidad.

\section{Método}

\section{Participantes}

Los participantes fueron 638 adolescentes (318 chicas), de 12 (240), 13 (197) y 14 años (201), $(M=12.93$; $D T=.82)$, de 4 Institutos de Educación Secundaria del extrarradio de Madrid con similares características socio-demográficas. No hubo diferencias en la distribución del sexo en las distintas edades $\left(\chi^{2}=1.417, p=.49\right)$. La participación fue voluntaria. Los directores de los centros autorizaron el estudio después de obtener el permiso de los padres.

\section{Instrumentos}

Matutinidad-Vespertinidad.- Se utilizó la escala de matutinidad-vespertinidad para niños, Morningness Eveningness Scale for Children, MESC (Carskadon et al., 1993). Está formada por 10 preguntas en las que se evalúa el horario de preferencia para realizar diversas actividades como son un examen, deporte o la hora ideal de acostarse y levantarse, entre otras. Cada uno de los ítems tiene 4 ó 5 opciones de respuesta. El rango de puntuaciones oscila desde 10 (vespertinidad) a 43 (matutinidad). La escala ha sido adaptada y validada al español con buenos indicadores psicométricos (Díaz-Morales y Gutiérrez, 2008). En el presente estudio la fiabilidad fue $\alpha=$ .71 .

Ansiedad.- El Inventario de Ansiedad Estado-Rasgo para Niños (STAIC; Spielberger, Edwards, Lushene, Montuori y Platzek, 1973; Spielberger et al., 1990) consiste en dos escalas de 20 ítems que evalúan la ansiedad estado y rasgo. La ansiedad estado evalúa cómo se siente la persona en un momento o situación determinados. La ansiedad rasgo evalúa cómo se siente habitualmente. En este estudio solamente se utilizó la escala de ansiedad rasgo, su fiabilidad fue $\alpha=.86$.

\section{Procedimiento}

Todos los participantes fueron evaluados de forma colectiva en su propio aula y en horario escolar (8:30-14:30) por evaluadores entrenados. Se utilizaron el Análisis de Varianza (ANOVA) y la correlación de Pearson como técnicas de análisis de datos. Como estadístico de medida del tamaño del efecto se utilizó el estadístico $\eta_{p}^{2}$ corregida (Huberty, 2002).
Los análisis se realizaron con el paquete de programas estadísticos SPSS-X (versión 15).

\section{Resultados}

La puntuación media en M-V fue 26.0 (DT = 4.44). La distribución de frecuencias no mostró asimetría (valor $=.12$, error $=.07)$ ni curtosis (valor $=.03$, error $=.14$ ). Se establecieron como puntos de corte los percentiles 25-75 (correspondientes a las puntuaciones 22/28 del MESC) para distinguir entre matutinos, intermedios y vespertinos. Con el objetivo de detectar las diferencias de sexo y edad en $\mathrm{M}-\mathrm{V}$ se realizó un ANOVA considerando la M-V como variable dependiente y el sexo y la edad $(12,13$ y 14 años) como factores de agrupación. Los chicos mostraron una mayor matutinidad comparados con las chicas $(M=26.72$ vs. $M=25.28)$, $F=16.72, p<.001, \eta_{p}^{2}=.02$. La matutinidad disminuyó a medida que aumentó la edad desde los $12(M=27.14)$, a los $13(M=25.62)$ y 14 años $(M=25.01), F=13.43, p<.001$, $\eta_{p}^{2}=.04$. Las pruebas post-hoc de Bonferroni indicaron que los adolescentes de 12 años obtenían una mayor puntuación en matutinidad que los de 13 ( $p<.01)$ y 14 años $(p<.001)$ (véase Figura 1). El efecto de interacción sexo*edad fue no significativo, $F=1.25, p=.28$. La correlación de Pearson indicó que la matutinidad disminuyó con la edad $(r=-.20, p$ $<.01)$, siendo esta disminución menor en chicos $(r=-.13, p$ $<.01)$, que en chicas $(r=-.25, p<.01)$.

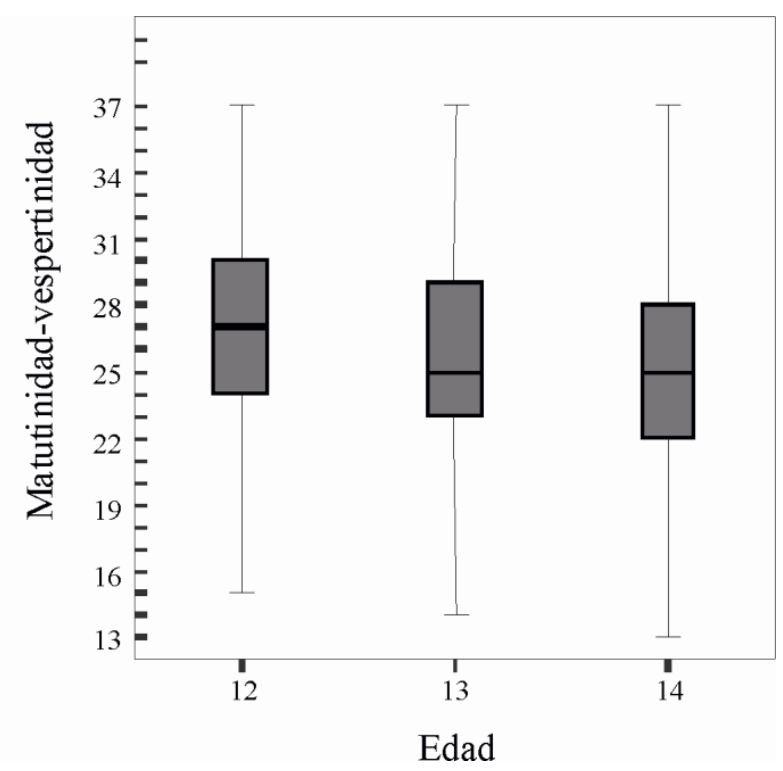

Figura 1. Puntuaciones en matutinidad-vespertinidad según edad.

La puntuación media en la escala de ansiedad rasgo fue $33.44(D T=6.95)$. La distribución de frecuencias mostró cierta asimetría positiva (valor $=.64$, error $=.09$ ) que se corrigió mediante la aplicación de la función raíz cuadrada (valor corregido $=.36$, error $=.09)$. Se realizó un ANOVA considerando la ansiedad rasgo como variable dependiente y el sexo, la edad y el cronotipo (matutino, intermedio y vesper- 
tino) como factores de agrupación. Los chicos mostraron una puntuación promedio en ansiedad rasgo menor que las chicas $(M=31.88$ vs. $M=35.01), F=28.28, p<.001, \eta_{p}{ }^{2}=$ .04. No hubo diferencias de edad en ansiedad rasgo, $F=.08$, $p=.92$. Los adolescentes matutinos obtuvieron una puntuación en ansiedad rasgo menor $(M=31.88)$ que los interme$\operatorname{dios}(M=33.59)$ y los vespertinos $(M=35.27), F=3.61, p$ $<.05, \eta_{p}^{2}=.01$. Las comparaciones post-hoc mediante la prueba de Bonferroni indicaron que los matutinos obtuvieron una puntuación en ansiedad rasgo menor que los intermedios $(p<.05)$ y los vespertinos $(p<.001)$. Los efectos de interacción entre el sexo y la edad $(F=.64, p=.52)$, el sexo y el cronotipo $(F=1.14, p=.31)$, la edad y el cronotipo $(F=$ $1.63, p=.16)$ y el sexo, la edad y el cronotipo $(F=2.01, p=$ .09) fueron no significativos. Por último, respecto a la relación entre la ansiedad rasgo y la M-V, la correlación de Pearson indicó una mayor ansiedad rasgo a medida que disminuía la matutinidad $(r=-.19, p<.01)$, tanto en los chicos $(r=-$ $.13, p<.01)$, como en las chicas $(r=-.18, p<.01)$.

\section{Discusión}

En este trabajo se analizó la relación entre $\mathrm{M}-\mathrm{V}$ y ansiedad rasgo en los primeros años de la adolescencia. La matutinidad disminuyó con la edad (Carskadon et al., 1993; DíazMorales y Randler, 2008; Kim, Dueker, Hasher y Goldstein, 2002). Los adolescentes de este estudio mostraron una tendencia hacia la vespertinidad mayor que la encontrada en otros países (véase Randler, 2008). Dado que el estudio se ha centrado en los primeros años de la adolescencia y, por tanto, en un rango de edad restringido (de 12 a 14 años), la mayor tendencia hacia la vespertinidad podría deberse a diferentes factores (veáse Díaz-Morales y Randler, 2008) como la latitud, el clima y distintos aspectos sociales y culturales relacionados con el uso del tiempo (Randler, 2008; Roenneberg, Kumar y Merrow, 2007).

Aunque varios estudios no encontraron diferencias de sexo en este grupo de edad (Carskadon et al., 1993; Kim et al., 2002), las chicas mostraron una mayor vespertinidad, en línea con la tendencia de estudios previos con muestras españolas (Delgado et al., 2012). Las diferencias de sexo podrían deberse, al menos en parte, a que las chicas tienen un desarrollo puberal más temprano y a influencias sociales y culturales (Park, Matsumoto, Seo, Shinkoda y Park, 1997). En relación al desarrollo puberal, Hagenauer, Perryman, Lee y Carskadon (2009) indicaron que las chicas muestran demora en el ciclo vigilia/sueño hasta un año antes que los chicos en paralelo al desarrollo puberal. Es posible que las chicas, a la misma edad que los chicos, sean más vespertinas puesto que se espera un desarrollo puberal más avanzado (Susman et al., 2007). En este sentido, futuros estudios podrían considerar el efecto del desarrollo puberal en la relación M-V y ansiedad. Por otro lado, los sincronizadores sociales influyen en el cronotipo (Roenneberg et al., 2007) de modo que el ambiente escolar, familiar y social puede explicar parte de las dife- rencias de género en la $\mathrm{M}-\mathrm{V}$ puesto que la socialización es distinta para los chicos que para las chicas. Por ejemplo, pueden diferir en la exposición a la luz solar, relacionada con la tendencia a la matutinidad, que es posible que se dé en mayor medida en los chicos por el hecho de que hacen deporte al aire libre con más frecuencia o tienen regímenes horarios más flexibles que las chicas (Gaina et al., 2006; Harada, Morisane y Takeuchi, 2002). Sin embargo, hasta el momento, poco es conocido sobre cómo estos factores interactúan para determinar el cronotipo individual (Schmidt y Randler, 2010).

Como se esperaba, las chicas obtuvieron una mayor puntuación en ansiedad rasgo. Carrasco y Espinar (2008) señalaron que tanto los aspectos genéticos como las diferencias en el proceso de socialización desde el nacimiento en función del sexo constituyen aspectos clave para explicar la mayor prevalencia de trastornos de ansiedad en las mujeres desde la infancia. En esta línea, los factores biológicos y sociales están asociados también a las características de personalidad (Bonilla-Campos y Martínez-Benlloch, 1999), por lo que es esperable que las chicas, además de por los factores comentados, muestren una mayor ansiedad rasgo debido a que al ser más vespertinas tendrán más dificultades para enfrentarse adecuadamente a las demandas escolares matutinas.

La relación entre la vespertinidad y una elevada ansiedad rasgo podría estar modulada por las hormonas gonadales que afectan tanto al reloj biológico (Hagenauer et al., 2009) como a la ansiedad (Reardon, Leen-Feldner y Hayward, 2009). En este sentido, la edad parece ser un factor importante que modifica las relaciones entre los cronotipos y la ansiedad rasgo. Por otro lado, puesto que el hipotálamo juega un papel central tanto en el desarrollo de la personalidad como en el sistema circadiano ambos podrían estar relacionados (Muro et al., 2009; Zuckerman, 1991). Así, la producción de monoaminas hipotalámicas, hormonas tales como melatonina o cortisol, y la responsividad a la sincronización del ciclo luzoscuridad podrían subyacer a las diferencias de personalidad entre los cronotipos (Hogben, Ellis, Archer y von Schantz, 2007).

Por último, este estudio presenta algunas limitaciones. Por ejemplo, no se tuvieron en cuenta las condiciones de vida ni familiares de los adolescentes, no se midió el desarrollo puberal, asociado tanto al retraso en ciclo vigilia/sueño y la vespertinidad (Carskadon et al., 1993; Hagenauer et al., 2009) como a la ansiedad (Reardon et al., 2009), ni se controló si los participantes tenían algún trastorno psicológico. Los resultados de este trabajo apuntan a que la regularidad en los hábitos de vigilia-sueño (Wittmann et al., 2006) y el desarrollo puberal podrían ser factores importantes que deben ser tenidos en cuenta en futuras investigaciones sobre cronotipo y ansiedad.

Agradecimientos.- Parte de este estudio ha sido financiado por el Plan Nacional I+D+I del Ministerio de Innovación y Ciencia (Ref: PSI2008-04086/PSIC). 


\section{Referencias}

Adan, A. (2010). Ritmicidad circadiana y adicción. Adicciones, 22, 5-10.

Adan, A., Fabbri, M., Natale, V. y Prat, G. (2006). Sleep Beliefs Scale (SBS) and circadian typology. Journal of Sleep Research, 15, 125-132.

Adan, A., Natale, V. y Caci, H. (2008). Cognitive strategies and circadian typology. En Léglise A.L. (ed.), Progress in circadian rbythm research (pp. 141-161). New York: Nova Science Publisher Inc.

Alonso, J., Angermeyer, M. C., Bernert, S., Bruffaerts, R., Brugha, T. S., Bryson, H., de Girolamo, G., de Graaf, R., Demyttenaere, K., Gasquet, I., Haro, J. M., Katz, S. J., Kessler, R. C., Kovess, V., Lépine, J. P., Ormel, J., Polidori, G., Russo, L. J. y Vilagut, G. (2004). Prevalence of mental disorders in Europe: Results from the European study of the epidemiology of mental disorders (ESEMeD) project. Acta Psychiatrica Scandinavica, 109, 21-27.

Andershed, A. K. (2005). In sync with adolescence: The role of morningness-eveningness in development. New York: Springer.

Benca, R., Duncan, M. J., Frank, E., McClung, C., Nelson, R. J. y Vicentic, A. (2009). Biological rhythms, higher brain function, and behavior: Gaps, opportunities, and challenges. Brain Research Reviews, 62, 57-70.

Bonilla-Campos, A. y Martínez-Benlloch, I. (1999). Relaciones sexo/género y cultura. En Sánchez-Cánovas, J. y Sánchez-López, M.P. (eds.), Psicología de la diversidad bumana (pp. 381-416). Madrid: Centro de Estudios Ramón Areces, D. L.

Carrasco, I. y Espinar, I. (2008). Trastornos de ansiedad y género. Mente y Cerebro, $31,12-21$

Carskadon, M. A., Vieira, C. y Acebo, C. (1993). Association between puberty and delayed phase preference. Sleep, 16, 258-262.

Delgado, P., Díaz-Morales, J. F., Escribano, C., Collado, M. J. y Randler, C. (2012). Morningness-eveningness and health-related quality of life among adolescents. Spanish Journal of Psychology, 15, 613-623.

Díaz-Morales, J. F. (2007). Morning and evening-types: Exploring their personality styles. Personality and Individual Differences, 43, 769-778.

Díaz-Morales, J. F., Delgado, P., Escribano, C., Collado, M. J. y Randler, C. (2011). Sleep beliefs and chronotype among adolescents: The effect of a sleep education program. Biological Rhythms Research, 43, 397-412.

Díaz-Morales, J. F. y Gutiérrez, M. (2008). Morningness-eveningness in adolescents. The Spanish Journal of Psychology, 11, 201-206.

Díaz-Morales, J. F. y Randler, C. (2008). Morningness-Eveningness among German and Spanish adolescents 12-18-years-old adolescents. European Psychologist, 13, 214-221.

Díaz-Morales, J. F. y Sánchez-López, M. P. (2008). Morningness-eveningness and anxiety among adults: A matter of sex/gender? Personality and Individual Differences, 44, 1391-1401.

Digdon, N. L. (2010). Circadian preference and college students' beliefs about sleep education. Chronobiology International, 27, 297-317.

Escribano, C., Díaz-Morales, J. F., Delgado, P. y Collado, M. J. (2012). Morningness/eveningness and school performance among Spanish adolescents: Further evidence. Learning and Individual Differences, 22, 409-413.

Fabbri, M., Antonietti, A., Giorgetti, M., Tonetti, L. y Natale, V. (2007). Circadian typology and style of thinking. Learning and Individual Differences, 17, $175-180$.

Gaina, A., Sekine, M., Kanayama, H., Takaski, Y., Hu, L., Sengoku, K. y Kagamimori, S. (2006). Morning-evening preference: Sleep pattern spectrum and lifestyle habits among Japanese junior high school pupils. Chronobiology International, 23, 607-621.

Gau, S. S-F., Shang, C-Y., Merikangas, K. R., Chiu, Y-N., Soong, W-T. y Cheng, A. T-A. (2007). Association between morningness-eveningness and behavioral/emotional problems among adolescents. Journal of Biological Rhythms, 22, 268-274.

Giannotti, F., Cortesi, F., Sebastiani, T. y Ottaviano, S. (2002). Circadian preference, sleep and daytime behaviour in adolescence. Journal of Sleep Research, 11, 191-199.

Hagenauer, M. H., Perryman, J. I., Lee, T. M. y Carskadon, M. A. (2009). Adolescent changes in the homeostatic and circadian regulation of sleep. Developmental Neuroscience, 31, 276-284

Harada, T., Morisane, H. y Takeuchi, H. (2002). Effect of daytime light conditions on sleep habits and morningness-eveningness preference of Japanese students aged 12-15 years. Psychiatry and Clinical Neurosciences, 56, 225-226.
Hogben, A. L., Ellis, J., Archer, S. N. y von Schantz, M. (2007). Conscientiousness is a predictor of diurnal preference. Chronobiology International, 24, 1249 1254

Huberty, C. J. (2002). A history of effect sizes indices. Educational Psychological Measurement, 62, 227-240.

Kalsbeek, A., Scheer, F. A., Perreau-Lenz, S., La Fleur, S. E., Yi, C-X., Fliers, E. y Buijs, R. M. (2011). Circadian disruption and SCN control of energy metabolism. FEBS Letters, 585, 1412-1426.

Kim, S., Dueker, G. L., Hasher, L. y Goldstein, D. (2002). Children's time of day preference: Age, gender and ethnic differences. Personality and Individual Differences, 33, 1083-1090.

Kohyama, J. (2009). A newly proposed disease condition produced by light exposure during night: Asynchronization. Brain \& Development, 31, 255-273.

Mecacci, L. y Rocchetti, G. (1998). Morning and evening types: Stress-related personality aspects. Personality and Individual Differences, 25, 537-542.

Monk, T. H., Buysse, D. K., Potts, J. M., DeGrazia, J. N. y Kupfer, D. J. (2004). Morningness-eveningness and lifestyle regularity. Chronobiology International, $21,435-443$.

Muro, A., Gomà-i-Freixanet, M. y Adan, A. (2009). Morningness-eveningness, sex, and the alternative five factor model of personality. Chronobiology International, 26, 1235-1248.

Negriff, S. y Dorn, L. D. (2009). Morningness-eveningness and menstrual symptoms in adolescent girls. Journal of Psychosomatic Research, 67, 169-172.

Pabst, S. R., Negriff, S., Dorn, L. D., Susman, E. J. y Huang, B. (2009). Depression and anxiety in adolescent females: The impact of sleep preference and body mass index. The Journal of Adolescent Health, 44, 554-560.

Park, Y. M., Matsumoto, K., Seo, Y. J., Shinkoda, H. y Park, K. P. (1997). Scores on morningness-eveningness and sleep habits of Korean students, Japanese students, and Japanese workers. Perceptual and Motor Skills, 85, 143-154.

Randler, C. (2008). Morningness-eveningness comparison in adolescents from different countries around the world. Chronobiology International, 25, 1017 1028.

Randler, C. (2011). Association between morningness-eveningness and mental and physical health in adolescents. Psychology, Health and Medicine, 16, 29-38.

Randler, C. y Saliger, L. (2011). Relationship between morningness-eveningness and temperament and character dimensions in adolescents. Personality and Individual Differences, 50, 148-152.

Reardon, L. E., Leen-Feldner, E. W. y Hayward, C. (2009). A critical review of the empirical literature on the relation between anxiety and puberty. Clinical Psychology Review, 29, 1-23.

Roenneberg, T., Kumar, C. J. y Merrow, M. (2007). The human circadian clock entrains to sun time. Current Biology, 17, 44-45.

Sandín, B. (1997). Ansiedad, miedos y fobias en niños y adolescentes. Madrid: Dykinson.

Schmidt, S. y Randler, C. (2010). Morningness-eveningness and eating disorders in a sample of adolescent girls. Journal of Individual Differences, 31, 38-45.

Spielberger, C. D., Edwards, C. D., Lushene, R. E., Montuori, J. y Platzek, D. (1973). STAIC Preliminary Manual. Palo Alto, CA: Consulting Psychologists Press.

Spielberger, C. D., Edwards, C. D. y Lushene, R. E. (1990). Manual del Cuestionario de Autoevaluación Ansiedad Estado-Rasgo en Niños (STAIC). Madrid: TEA.

Susman, E. J., Dockray, S., Dorn, L. D., Schiefelbein, L. V., Herwehe, S. y Heaton, J. A. (2007). Morningness/eveningness, morning-to-afternoon cortisol ratio, and antisocial behavior problems during puberty. Developmental Psychology, 43, 811-822.

Tankova, I., Adan, A. y Buela-Casal, G. (1994). Circadian typology and individual differences. A review. Personality and Individual Differences, 16, 671-84.

Wittmann, M., Dinich, J., Merrow, M. y Roenneberg, T. (2006). Social jetlag: Misalignment of biological and social time. Chronobiology International, 23, $497-$ 509.

Zuckerman, M. (1991). Psychobiology of personality. Cambridge: Cambridge University Press.

(Articulo recibido: 14-11-2011, revisado: 05-03-2012, aceptado: 05-03-2012) 